New York University

Washington Square College

Mathematics Research Group

Research Report No. TW-9

\title{
AMPLIFICATION OF THE TRAVELING WAVE TUBE \\ by
}

Bernard Friedman

The research reported in this document has been made possible through support and sponsorship extended by the Cambridge Field Station, AMC, U. S. Air Force, under Contract No. AF-19 (122)-1. It is published for technical information only and does not represent recommendations or conclusions of the sponsoring agency.

February, 1949 


\author{
Hew York University \\ Washington Square Colle ู้อ \\ Matinematics Research Group \\ Research Report Lo.Ti-9 \\ under \\ Contract No. AF-13(122)-1
}

AVPIEICATION OF THE TRAVELING WAVE TUBA

by

Bernard Friedman

iritten by:

Bermand Fridaman

Berrara Ariedman

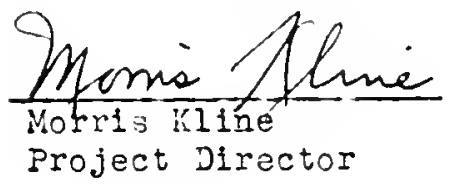

Title page

16 numiered pages

4 trapis

February, 1949 

Article

Abstract

Introduction $\underline{\text { Page }}$

i

1

2

4

10

12

\section{Abstract}

This paper presents a. Inethod for solving the natching equation fiven by chu and Jackson "Field Theory of Travelinf Wave Mubes", iProc. I.R.E., Vol. 36, July, 1948, D. 853). The solution of this equation gives modes of the helix with the electron beam and thus determines the amplification factor. The essential idea is to consider the modes as a perturbation of the modes produced by the cold hellx. The dependence of the amplification factor on the paraseters of the tube can be explicitly deteruined. one surprising result is that the tube will not amplify for too larje values of the D.C. electron beam. 
•

- 


\section{Introduction}

In a recent raper, Chu and Jackson ${ }^{1}$ have tnvestigated the modes of an 1dealtzed traveling wave tube. They show that the propagation constant $Y_{n}$ assoctated with the nth mode satisfles a complicated transcendental equation. By making some rough aprioximations, the equation reduces to a cublc whose solutions can be found. However, thetr method is subject to the criticisu that in order to perform the reduction, all the parameters of the probler must be fixed. This weans that when any of the paraneters is chanjed, the whole procedure for solving the equation must be repeated. As a result, the effect upon the propafation constants of a chane in parameters can not be determined.

In this report a simpler and more exact method is presented for solvine the transcendental equation given by Chu and Jackscn. The method yields explicltly the manner in which the yropagation constants derend on the parameters so that the effect of a change of parameters can be readily calculated. In particular the maximur amplification and the cand vidt of the tube may be found.

The theory and method presented here have been apolied to some cases of the travaling wave tube. It has keen difficult to compare the theoretical recults with the experimental ones jecause the racius of the electron bean is very difflcult to measure. It is hoped that this difficulty may be overcone so that an adeouate comparison with expertment could be made.

In section 1, we have civen a sumary of that vart of the naper of Chu and Jeckson wicn yielas the basic natchine equation.

In section?, the basic ecuation is reduced to a cublc enuation. The coefficients of the cubic equation ore explicitly given in terms of the ceometry of ti.e tube and the ratio $\frac{I}{V / \sqrt{2}}$ of the electron bean. ( $I$ is the d.c. corrent and $V$ is the voltace of the electron bear).

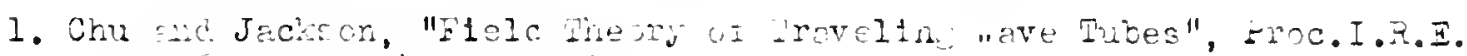
Vol. $36, J u i r 1948 \div .853)$. 
.

. 
In section 3, the question of maximum explification is investigated. A nethod for finding maximum amplification is given. From the theory of this section, graphs are given for maximum axplification in terms of the ratio $\frac{I}{V / 2}$. Graphs have also been constructed for the quantity $d_{0}$ in terms of $\frac{I}{V^{3 / 2}}$, where $d_{0}$ is the difference between the wave number for the electron beam and the wave number of the "cold" helix. In section 4, an investitration is made of the case vhere als is large. Asyuptotic formulas for the Bessel functions are used and the cubic equation of section 2 is reduced to a simpler cubic. The amplification of the wave, in this case, can be explicitly written dorm in terms of the narameters of the tuke. Also it is shown that the tube will not amplify for too large values of the d.c. current.

1. Summary of the principal result of Chu and Jackson.

In the paper by Chu and Jackson the actual helix is replaced by a losslegs helicel sheath of radius a and en infinitesmal thickness. The current flow alons the sheath is constrained to wove in a direction which wakes a constant antle $90^{\circ}-\theta$ with the axis of the helix. The force acting on tire electrons is restricted to that associated with the loneitudinal electric field only; and the electrons are assumed to have no initial transverse motion. It is also assumed that the electrons are confined within a cylinder of radius $b$ concentric with the helical sheath. The time-average beam current density is assured constant over the cross section.

Chu and Jackson tackle the problen as a boundary velue problem. Three regions are cefined: the region outside the cylinder, the region between the cylinder and the stream of electrons and iinally the region occupied by the electrons, that is, $r<b$. Field componente for $E$ and $H$ are deduced from liaxwell's Equations and the fields are matched on the two bcundaries. If the fields cre as sured to be circularly symetric 


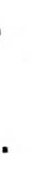


and vary with $e^{i \omega t-\gamma z}$, the transcendental equation for the propagation constant $\gamma$ is found to be

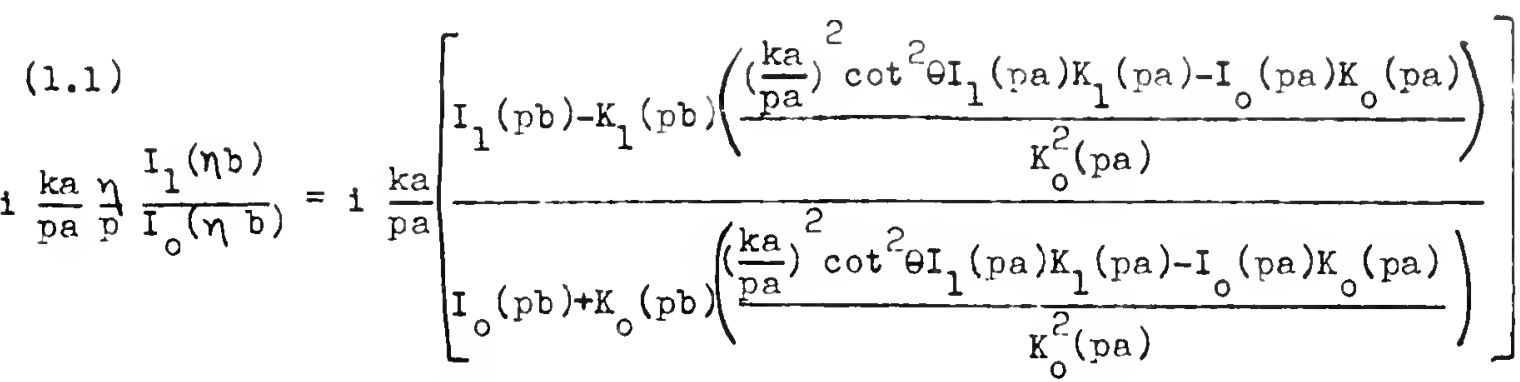

where $I_{0}(x), I_{1}(x), K_{0}(x)$ and $K_{1}(x)$ are modified Bessel functions: and where

$$
k^{2}=\omega^{2} \varepsilon \mu, k=\frac{2 \pi}{\lambda}=\frac{\omega}{c}
$$$$
p^{2}=-\left(\gamma^{2}+k^{2}\right)
$$

$$
\eta^{2}=p^{2}\left[1+\frac{\frac{e}{m} I}{\pi b^{2} \varepsilon v_{0}^{3}\left(1 \frac{\omega}{v_{0}}-\gamma\right)^{2}}\right]
$$

$$
\frac{e}{m}=\text { ratio of charge of electron to mass }
$$

$$
v_{0}=\text { average value of the electron bean velocity. }
$$

$$
I=\text { d.c. beam current }
$$

In order to discuss the behavior of the tube, 玉o. (1.1) must be solved for $p$. A real root of this equation corresnonds to a progressive wave while a comlex root with a positive imaginary part indicates that the correspondin wave will be amplified. Chu and Jackson Indicate a method for its solution. They plot the right hand side of Eq. (1.1) for a narticular chofce of parameters. Its graph suggests that it can be replaced by the function

$$
\text { R.H.S. }=1 k a C \frac{p-p^{\prime}}{p-p^{\prime 1}} \text {. }
$$

The left hand side of En. (1.1) is expanded into a pover series in $\frac{\eta}{p}$ and the first term

$$
\text { L.H.S. }=1 \frac{\mathrm{ka}}{2} \frac{\eta^{2}}{2} \frac{\mathrm{b}}{2}
$$
Vo. 36, July 1948, pp.853-863, Equations $(20),(25)$. 
. 
only is retained. Using Eqs. $(1.4),(1.8)$ and (1.9) there results the equation

$$
\text { (1.10) } \frac{b}{a} I+\frac{\frac{e}{m} I}{\pi b^{2} \varepsilon{ }_{0}^{3}\left(i \frac{\omega}{v_{0}}-\gamma\right)^{2}}=2 C\left(\frac{\gamma-i \beta^{\prime}}{\gamma-i \beta^{\prime \prime}}\right) \text {, }
$$

where $p \cong-i \gamma, p^{\prime}=i \beta^{\prime}, p^{\prime \prime}=i \beta^{\prime \prime}$. The equation $p \cong-i \gamma$ follows

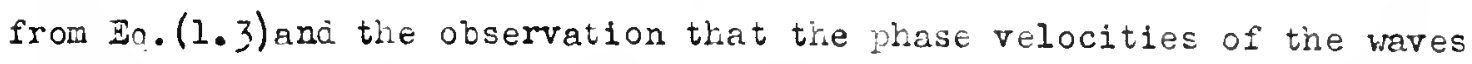
are unch less than that of light. iq. (1.10) is the equation derived by Chu and Jackson. Its derivation, as has been stated in the introduction, depends on fixing all the parameters of the equation(1.1.)

2. Approximate Solution of the Fundamental Equation.

In this section a metiod will be given for solving Ea. (1.1). Rewrite Eo. (1.1) in the form

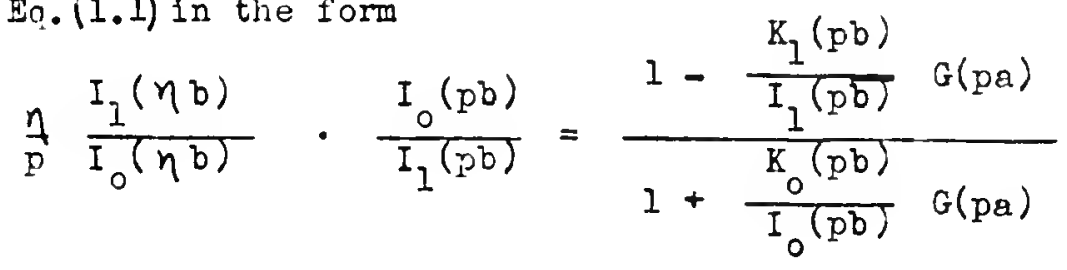

where

(2.?) $G(p a)=\frac{I_{0}(p a)}{K_{0}(p a)}\left[\left(\frac{k a \cos \theta}{p a}\right)^{2} \frac{I_{1}(p a)}{I_{0}(p a)} \frac{K_{1}(p a)}{K_{0}(p a)}-1\right]$.

In the absence of an electron beam the modes are given by the equation

$$
G(p a)=0 \text {. }
$$

The crux of the present method is to consider the roots of Jq. (2.1) as being found by means ol a perturbation of the roots of aq. $(2.3)$.

Let $p_{0} a$ be the single root of $=0 \cdot(2.3)$ Put

$(2.4)$

$$
\mathrm{p}_{0} \mathrm{a}=\mathrm{x}_{0}, \mathrm{pa}=\mathrm{x}_{0}+\mathrm{y}
$$

where $y$ is ssumed to be snall compared to $x_{0}$. The right hand side of Eq. (2.1) may be written as 


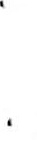


(2.5) R.H.S. $=1-\frac{G(p a)\left\{\frac{K_{1}(p b) I_{0}(p b)+K_{0}(p b) I_{1}(p b)}{I_{1}(p b) I_{0}(p b)}\right\}}{1+\frac{K_{0}(p b)}{I_{0}(p b)} G(p a)}$

$$
=1-\frac{\frac{G(p a)}{p b I_{1}(p b) I_{0}(p b)}}{1+\frac{K_{0}(p b)}{I_{0}(p b)} G(p a)} .
$$

where use has been made of the Vronskian relationship

(2.6) $\quad K_{1}(\underline{p b}) I_{0}(p b)+K_{0}(p b) I_{1}(p b)=\frac{1}{p b}$.

If now $G(p a)$ is expinded in a Iailor's series about $x_{0}$, there

results

(2.7) $\quad G(p a)=G\left(x_{0}+y\right)=G\left(x_{0}\right)+y G^{\prime}\left(x_{0}\right)+\ldots$

$$
\begin{aligned}
& =y G^{\prime}\left(x_{0}\right)+\ldots \\
& =y G^{\prime}\left(x_{0}\right), \text { approximately. }
\end{aligned}
$$

Replacing G(pa) in En. (2.5) by its value, it is found that

$(2.8)$

$$
\text { x.H.S. }=1-\frac{\frac{y G^{\prime}\left(x_{0}\right)}{1+\frac{x_{0}\left(\frac{b}{a} x_{0}\right) I_{1}\left(\frac{b}{a} x_{0}\right)}{I_{0}\left(\frac{b}{a} x_{0}\right)}} G^{\prime}\left(x_{0}\right) y}{a}
$$




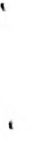

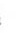


For the left hand side of the fundamental equation we proceed as follows. Expand the function $\frac{I_{I}(r x)}{I_{0}(r x)}$ in a Trylor's series in $r$ about $r=1$. we find that

$$
\begin{aligned}
& \text { (P. 3) } \frac{I_{1}(r x)}{I_{0}(r x)}=\frac{I_{1}(x)}{I_{0}(x)}+(r-1) x\left[\frac{I_{I}^{\prime}(x) I_{0}(x)-I_{1}(x) I_{0}^{\prime}(x)}{I_{0}^{2}(x)}\right]+\ldots \\
& =\frac{I_{1}(x)}{I_{0}(x)}+(r-1) x\left[\frac{I_{0}^{2}(x)-\frac{1}{x} I_{0}^{\prime}(x) I_{0}(x)-I_{1}^{2}(x)}{I_{0}^{2}(x)}\right] .
\end{aligned}
$$

Frun iq. $(2.9)$ it follows that

$$
\frac{I_{1}(r y)}{I_{0}(r y)} \frac{I_{0}(x)}{I_{1}(x)}=1+(r-1) x\left[\frac{I_{0}(x)}{I_{1}(x)}-\frac{I_{1}(x)}{I_{0}(x)}-\frac{1}{x}\right]
$$

If we let

$$
r=\frac{\eta}{p}
$$

the left hand side of the fundenentel equation is

$$
\begin{aligned}
\text { L.H.S. } & =\frac{\eta}{p}+\left(\frac{\eta}{p}-1\right) \frac{\eta}{p}\left[\frac{I_{0}(p b)}{I_{1}(p b)}-\frac{I_{1}(p b)}{I_{0}(p b)}-\frac{1}{p b}\right] p b \\
& =\frac{\eta}{p}+\left(\frac{\eta}{p}-1\right) \frac{\eta}{p}\left[\frac{I_{0}\left(\frac{b}{a} x_{0}\right)}{I_{1}\left(\frac{b}{a} x_{0}\right)}-\frac{I_{1}\left(\frac{b}{a} x_{0}\right)}{I_{0}\left(\frac{b}{a} x_{0}\right)}-\frac{1}{\frac{b}{a} x_{0}}\right] \frac{b}{a} x_{0} \\
& =\frac{\eta}{p}+\left(\frac{\eta}{p}-1\right) \frac{\eta}{p} \delta .
\end{aligned}
$$

where

$(2.11) \delta=\left[\frac{I_{0}\left(\frac{b}{a} x_{0}\right)}{I_{I}\left(\frac{b}{a} x_{0}\right)}-\frac{I_{1}\left(\frac{b}{a} x_{0}\right)}{I_{0}\left(\frac{b}{a} x_{0}\right)}-\frac{1}{\frac{b}{a} x_{0}}\right] \frac{b}{a} x_{0}$ 


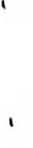


Assuming $\frac{\eta}{p}=1$, the Eq. (2.11) becomes

(2.12) L.H.S. $=\frac{n}{p}+\left(\frac{n}{p}-1\right) \delta$.

Equatini 3. (2.8) and Eq. (2.12) the bastc equation is replaced by

(2.13) $\frac{\eta}{p}+\left(\frac{n}{p}-1\right) \delta=1-\frac{\frac{y G^{\prime}\left(x_{0}\right)}{\frac{b}{a} x_{0} I_{0}\left(\frac{b}{a} x_{0}\right) I_{1}\left(\frac{b}{a} x_{0}\right)}}{1+\frac{K_{0}\left(\frac{b}{a} x_{0}\right)}{I_{0}\left(\frac{b}{a} x_{0}\right)} G^{\prime}\left(x_{0}\right) y}$,

or

$(2.24)\left(\frac{n}{p}-I\right)(1+\delta)=-\frac{\frac{y G^{\prime}\left(x_{0}\right)}{\frac{b}{a} x_{0} I_{0}\left(\frac{b}{a} x_{0}\right) I_{1}\left(\frac{b}{a} x_{0}\right)}}{I+\frac{K_{0}\left(\frac{b}{a} x_{0}\right)}{I_{0}\left(\frac{b}{a} x_{0}\right)} G^{\prime}\left(x_{0}\right)}$.

We know, however, that

$(1.4)$

$$
\frac{\eta^{2}}{\underline{n}^{2}}=I+\frac{\frac{e}{\mathrm{n}} I}{\pi b^{2} \varepsilon v_{0}^{3}\left(i \frac{\omega}{v_{0}}-\gamma\right)^{2}}
$$

But tine phase velocities of tine bean are unch less than that of light. thus (see E.

$$
\gamma=i p
$$


$-8-$

Hence El. (1.4) is

$$
\frac{\eta^{2}}{p^{2}}=1-\frac{\frac{\theta}{m} I}{\pi b^{2} \varepsilon v_{0}^{3}\left(\frac{\omega}{0}-p\right)^{2}}
$$

$$
\text { (2.16) } \begin{aligned}
& =1-\frac{\frac{e}{m} I}{\pi \frac{b^{2}}{a^{2}} \varepsilon v_{0}^{3}\left(\frac{\omega}{v_{0}} a-x_{0}+\pi_{0}-a p\right)^{2}} \\
& =1-\frac{\frac{e}{m} I}{\pi \frac{b^{2}}{a^{2}} v_{0}^{3}\left(d_{0}-y\right)^{2}} .
\end{aligned}
$$

where

$$
\text { (2.17) } \quad d_{0}=\frac{\omega}{v_{0}} a-\pi_{0} \text {. }
$$

Hence

$$
\frac{\eta}{p}=1-\frac{\frac{e}{m} I}{2 \pi \frac{b^{2}}{a^{2}} \varepsilon v \int_{0}^{3}\left(d_{0}-y\right)^{2}}
$$

$(2.18)$

$$
\begin{aligned}
& =1-\frac{1}{\pi \varepsilon 4 \sqrt{2}\left(\frac{e}{m}\right)^{1 / 2} \frac{b^{2}}{a^{2}}} \frac{I}{v^{3 / 2}} \cdot \frac{1}{\left(d_{0}-y\right)^{2}} \\
& =1-\frac{w}{b^{2} / a^{2}} \frac{1}{\left(d_{0}-y\right)^{2}}
\end{aligned}
$$



where

$$
v=\frac{\frac{e}{m}}{\pi \frac{b^{2}}{a^{2}}} \frac{I}{v_{0}^{3}}=\frac{1}{\pi \varepsilon 2 \sqrt{2}\left(\frac{e}{m}\right)^{1 / 2} \frac{b^{2}}{a^{2}}} \frac{I}{v^{3 / 2}}
$$

since $1 / 2 m v_{0}^{2}=\in V$. Combining Eqs. (2.18), (2.14) and (2.8), we get $(? .00) \quad y\left(y-d_{0}\right)^{2}-\xi(1+\xi y)=0$

where

(2.21) $\}=\frac{x_{0} I_{0}\left(\frac{b}{a} x_{0}\right) I_{1}\left(\frac{b}{a} x_{0}\right)(I+\delta) W}{2 \frac{b}{a} G^{\prime}\left(x_{0}\right)}$

(2. ?.?) $\xi=\frac{K_{0}\left(\frac{b}{a} x_{0}\right)}{I_{0}\left(\frac{b}{a} x_{0}\right)} G\left(x_{0}\right)$.

Enation (?.20) is the desired cubic onuation, $\zeta$ and $\xi$ are known aumtities denendin on the ceometry of the tube, and the voltage and current of the electron beam. In the case where the electron beam travels at slich a speed that it is in exact step with the wave traveling along the helix, we heve $d_{0}=0$. Thus $d_{0}$ mar be considered a measure of how far fron resonance the tube is.

The cubic equation (2.20) has real coefiticients. It has three real roots, or one real root and a pair of complex roots. The case of three raal roots (Y pure ina ineru) corresjonds to nodes wich are neither attenueted nor molitied. In the other case, however, the pair of cormolex conjueate rots ive rise on the one hand to an attenuated wave, on the otier fand on anjilier wave. The mount of attenuation and amolifica-

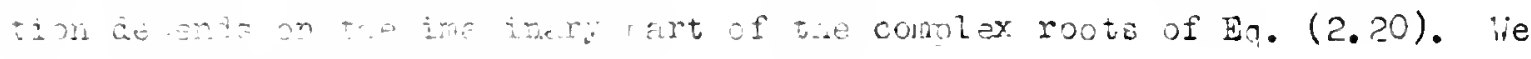
shall inveztibate the question in tre nert section. 
3. Maximula Amplification of the Tube.

The auestion of what values of the parometers give inaximun amplification will now be taken up. It will be shom that maximum anolification occurs for a value of $d_{0}>0$ so that the velocity of the electron beam should be slightly less than that of the wave down the tuive. But the maximum is so broid that $d_{0}=0$ will give armlification close enough to maximus.

Ve start vith our basic equation

$$
\text { (3.l) } \quad y\left(y-d_{0}\right)^{2}-\zeta(I+\xi y)=0
$$

ond pose the problem of determinin the value of $\mathrm{d}_{0}$ which will bive maxivim amplification.

Let

(3.2) $\quad y=z \xi^{1 / 3}, T=a_{0} / \xi^{1 / 3}, \rho=\xi \zeta^{1 / 3}$

then $E_{\eta}$. (3.1) becones

$$
z(z-\tau)^{2}-(z+\rho z)=0
$$

and we ask what value of $\tau$ will yieli the maximum imacinary part for a complex root of in. (3.3).

Let

(3.4)

$$
z=\alpha+1 \beta,
$$

then, siuce all coefficients are real, the imanary part of En. (3.3) may be written as

$$
\beta^{2}=3 \alpha^{2}-4 \alpha \tau+\tau^{2}-\rho
$$

Let $z_{0}$ be the real root of En. (3.3), then

$$
\text { (3.6) } \quad z_{0}+2 \alpha=2 \tau, \alpha=\tau-\frac{z_{0}}{2}
$$


-11-

Differentiating Eo. (3.5) with respect to $\tau$ yields

(3.7) $\frac{d \beta^{2}}{d \tau}=b \alpha \frac{d \alpha}{d \tau}-4 \tau \frac{d \alpha}{d \tau}-4 \alpha+2 \tau=0$

for a naximum, or

$$
\frac{d \alpha}{d \tau}=\frac{2 \alpha-\tau}{3 \alpha-2 \tau}=\frac{T-{ }_{0}{ }_{0}}{T-\frac{3}{2} z_{0}} .
$$

But

$(3.9)$

$$
\frac{d \alpha}{d \tau}=1-\frac{1}{2} \frac{d z_{0}}{d \tau}
$$

and

(3.1C) $3 z_{0}^{2} \frac{d z_{0}}{d T}-4 T z_{0} \frac{d z_{0}}{d \tau}+\left(T^{2}-\rho\right) \frac{d z}{d T}-2 z_{0}^{2}+2 T z_{0}=0$

so that

(3.11) $\frac{\mathrm{d} z_{0}}{\mathrm{~d} \tau}=\frac{2 z_{0}^{2}-2 \tau z_{0}}{3 z_{0}^{2}-4 T z_{0}+\tau^{2}-p}=2\left(1-\frac{d \alpha}{d \tau}\right)=-\frac{z_{0}}{\tau-\frac{3}{2} z_{0}}$.

En. (3.11) rejuces to

$$
2 T z_{0}=2 T^{2}+2 p .
$$

or

$$
z_{0}=\tau+\frac{\rho}{\tau}
$$

Usine the value of $z_{0}$ given by Er. (3.12) in Eo. (3.3) tives us an ecuation which reduces to $(3.1 .3)$

$$
\tau^{3}(1+\rho \tau)=\rho^{3}
$$


Wh. (3.13) is the solution to our problem. A graph of $T$ as a function of $\rho$ is given in Tigure 1.

$$
\text { Now use Eo. (3.12) in En. (3.5) to get }
$$

$$
\beta^{2}=\frac{1}{4}\left(\frac{3 \rho}{\tau}+\tau\right)\left(\frac{\rho}{\tau}-\tau\right)
$$

Eq. (3.14) provides the maximu imaginary part for a given $\tau$.

$$
\text { Finally, in terms of the original parameters, Eq. (3.13) }
$$

becomes

(3.15)

$$
\mathrm{d}_{0}^{3}\left(1+\xi \mathrm{d}_{0}\right)=\xi^{2} \xi^{2}
$$

and since the imaginary na"t of $\mathrm{y}$ is $\beta \zeta^{1 / 3}$, Eq. (3.14) vecomes

$$
\beta^{2} \zeta^{2 / 3}=\frac{1}{4}\left(\frac{3 \xi \zeta}{d_{0}}+d_{0}\right)\left(\frac{\xi \zeta}{d_{0}}-d_{0}\right) .
$$

4. Discussion for larse values of ka.

Furtier discussion of the problem is possible if it is assumed that ak is large, say about 3 or greater. The Eq. (1.1) again reduces to a cubic equation but since the Bessel functions may, in this case, be replaced by their asymptotic values, the coeficients $\xi$ and $\zeta$ of Iq. (2.20) are considerably simplified.

$$
\text { First, we note that En. (2.3) becomes }
$$

$$
\left(\frac{\mathrm{pa}}{\mathrm{ka}}\right)^{2} \tan ^{2} \theta=1 \text {, }
$$

so that

$$
x_{0}=k a \cot \theta
$$

Assume that the electron beam is in step with the wave, that is

$$
\text { (4.3) } \mathrm{d}=0=\frac{\omega_{\mathrm{a}}}{v_{0}}-\mathrm{x}_{0}=\frac{\omega_{\mathrm{a}}}{v_{0}}-\operatorname{akcot} \theta
$$

so that

$$
\nabla_{0}=c \tan \theta
$$


$-13-$

20. (2.20) reduces to

$(4.5)$

$$
y^{3}-\zeta \xi y-\zeta=0
$$

where for the sake of convenience we shall rewrite $\zeta$ and $\xi$. ire have

$$
\begin{aligned}
& \text { (4. c) } \quad S=\frac{x_{0} I_{0}\left(\frac{b}{a} x_{0}\right) I_{1}\left(\frac{b}{a} x_{0}\right)(I+\partial) W}{2 \frac{b}{a} G\left(x_{0}\right)} \\
& \text { (4.7) } \xi=\frac{K_{0}\left(\frac{b}{a} x_{0}\right)}{I_{0}\left(\frac{b}{a} x_{0}\right)} G^{\prime}\left(x_{0}\right),
\end{aligned}
$$

where

(4.8) $\quad i=\frac{1}{2 \sqrt{2} \sqrt{\frac{e}{m}} \frac{b^{2}}{a^{2}}} \cdot \frac{I}{v^{3 / 2}}=l \frac{I}{v^{3 / 2}}$ say,

and

$$
\begin{aligned}
& (1+9)(1+\delta)=\frac{b}{a} x_{0}\left[\frac{I_{0}\left(\frac{b}{a} x_{0}\right)}{I_{1}\left(\frac{b}{a} x_{0}\right)}-\frac{I_{1}\left(\frac{b}{a} x_{0}\right)}{I_{0}\left(\frac{b}{a} x_{0}\right)}\right] \text {. } \\
& \text { If use is made of the asymptotic formulas } \\
& \text { (4.12) } \quad I_{\nu}(x) \cong \frac{1}{\sqrt{2 \pi x}} e^{x}, K_{\nu}(x) \cong \sqrt{\frac{\pi}{2 x}} e^{-\pi}, \nu=0,1 \text {, } \\
& \text { the coefficients } \zeta, \zeta \xi \text { of } 1 \text {. } .(4.5) \text { reduce to } \\
& \text { (4.21) } \\
& \zeta=-\frac{\left(\frac{a}{n}\right)^{4} x_{0} l}{8 e^{-2 x_{0}\left(\frac{b}{a}-1\right)}} \frac{I}{v^{3 / 2}} \text {. } \\
& \text { (1.12). } \quad \zeta \xi=\frac{\left(\frac{a}{b}\right)^{4} l}{4} \frac{I}{v^{3 / 2}} \text {. }
\end{aligned}
$$



Hence Ë.

$$
\text { (4.13) } \quad \mathrm{Ay}^{3}-\mathrm{LA} \mathrm{Y}+\mathrm{L}=0 \text {, }
$$

where

$$
L=\left(\frac{a}{b}\right)^{2} \frac{\ell}{4} \frac{I}{V^{3 / 2}}
$$

$$
A=\frac{2}{x_{0}} e^{-2 x_{0}\left(\frac{b}{a}-i\right)}
$$

Put

$$
y=\left(\frac{L}{A}\right)^{1 / 3} u
$$

then Eq. (4.13) becomes

$$
u^{3}-3 u+1=0,
$$

where

$$
B=A^{2 / 3} L^{1 / 3}=\left(A^{2} L\right)^{1 / 3} .
$$

Eq. (4.3.7) will have inacinary roots if

$(4.19)$

$$
B<\frac{3}{\sqrt[3]{4}}
$$

The quantity $B_{1}$ however, contains the term $\left(\frac{I}{V 3 / 2}\right)^{1 / 3}$. Thus

If all other quantities are kept constant, we see that no amplification occurs if the above ratio becones too large.

Another consequence of Eq. (4.17) is as follows: Suppose
$(4 . ? .0)$
$B<1$, 

This imnlies that u is approximaiely 1. A better approximation is

(4.21)

$$
\begin{aligned}
u=-\sqrt[3]{1+B} & =-1-\frac{B}{3}+\ldots \\
& =-1-\frac{B}{3} .
\end{aligned}
$$

But if the real root of I0. (4.17) is given by EO. (4.21), the guadratic eouation yieldin the imaginary roots is given by

$(4.22)$

$$
u^{2}-u\left(1+\frac{B}{3}\right)+\left(1-\frac{B}{3}\right)=0,
$$

or

(4.23)

$$
u=\frac{1+\frac{B}{3} \pm \sqrt{-3+2 B}}{2}
$$

Hence the inafinary nart is

$$
\text { (4.-4) } \quad \operatorname{Im}= \pm \frac{\sqrt{3-23}}{2}= \pm \frac{\sqrt{3}}{2}\left(1-\frac{2}{3} B\right) .
$$

An exarple of the use of results of this section will be given. Let $x_{0}=k a \cot \theta=2.4 ; \quad \frac{b}{a}=.8, I=25 \mathrm{ma}, V=2500$. Then Da. $(4.3 .4),(4.15)$ becorne

$$
I=2.35 \times 10^{-3}, A=2.18
$$

Thus

$$
\left(\frac{I}{A}\right)^{1 / 3}=1.024 \times 10^{-1}, B=\left(A^{2} I\right)^{1 / 3} \equiv 2.2 \times 10^{-1},
$$

and $\frac{1}{2} \sqrt{3-23}=.80$. Hence Eo. (4.16) Eives for the imaginary part of the root oi sio. (4.13)

$$
\operatorname{Im} y=.0820 \text {. }
$$



This is in agreement with the graph in figure 3 for the values of the parameters $\frac{b}{a}=.8, h=2.4$ and $10^{6} \frac{I}{v^{3 / 2}}=0.2$. Since $d_{0}$ is small, the amplification could be comuted by the results of this section rather tiran those of section 3 .

Referrine back to the graphs in figures 2 and 3, it is seen that the curves for maximum armlification reach a maximum for $h=2.0$, $\frac{b}{a}=.8, h=2.5, \frac{b}{a}=.6$. An explanation of this is afforded by the work of the present section.

It is readily verified that if $\frac{b}{a}$ is fixed and if $0<\frac{b}{a}<1$, the function $\mathrm{A}$ of $\mathrm{EO}_{2}$. (4.5) has a minimum for $x_{0}=\frac{1}{2\left(1-\frac{b}{a}\right)}$. The quantity $\mathrm{L}$ of $\mathrm{Eq}$. $(4.14)$ is irdeperdent of $x_{0}$. Thus it is possible for, the roots of Eo. (4.13) to be the same for two different values of $x_{0}$. In the work of section 3 , the quantity $h$ is close to $x_{0}$.

This remark way have practical value for experiment since it indirates that the pitch angle should be so chosen that ka $\cot \theta=2.0$ for $\frac{b}{a}=.8$ and $\mathrm{ka} \cot \theta=0.5$ for $\frac{\mathrm{b}}{\mathrm{a}}=.6$. 



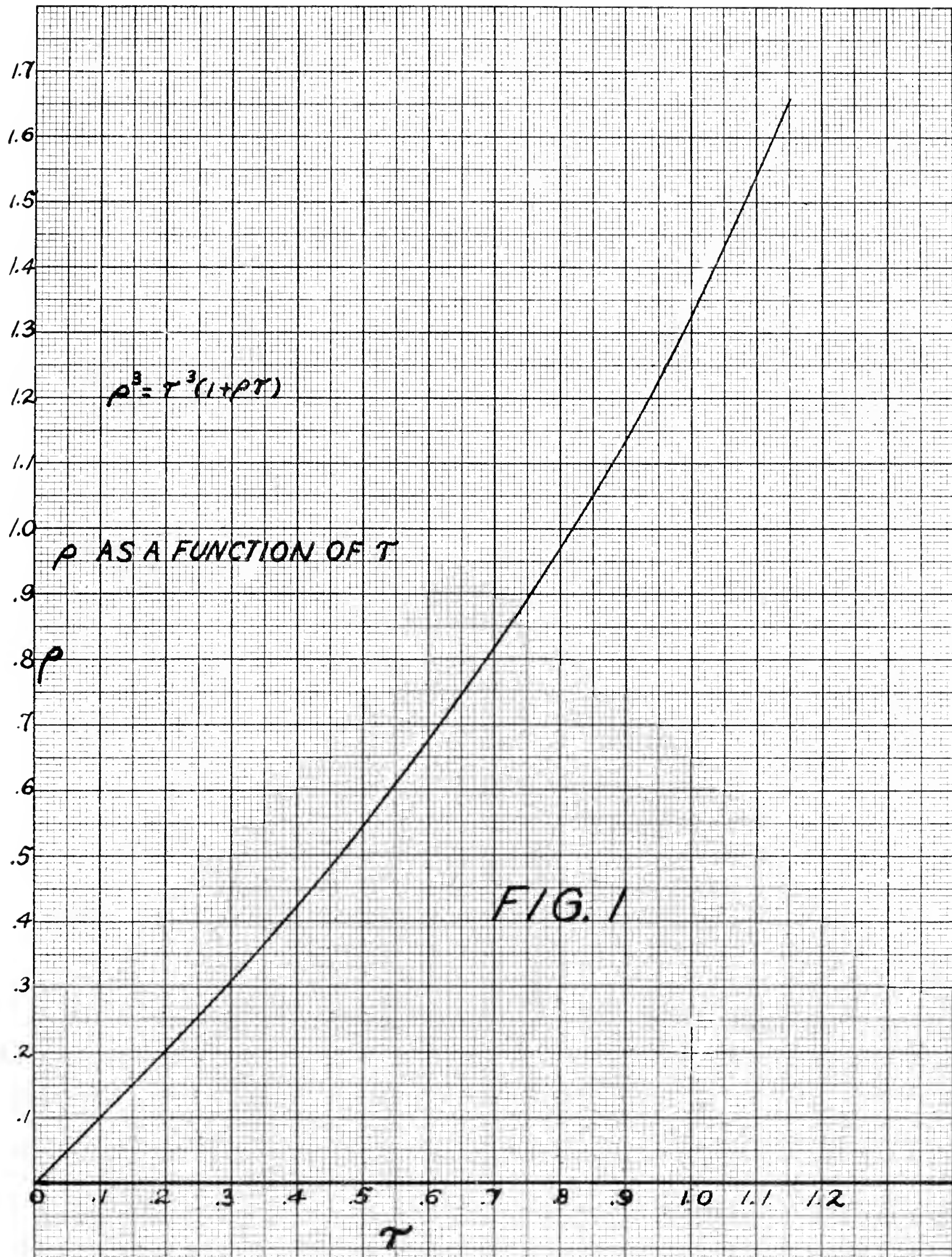



$.20-d 0$

.15

- d. A S A FUNCTION of $\frac{V^{6} I}{V^{3 / 2}}$

$\frac{b}{a}=\sigma$
$q_{0}=\frac{\rho c 0}{v_{0}}-x_{0}$

$h=a k \cot \theta$

.10

.05

$$
.10 .20 .30 \quad 40.50 \quad 60 \quad 70 \quad .80 .90 \quad 10
$$

.20 $\frac{10^{6} T}{V^{372}}$

.15 MAX AMR AS A FUNCTION OF $\frac{10^{6} f}{V^{3 / 2}}$

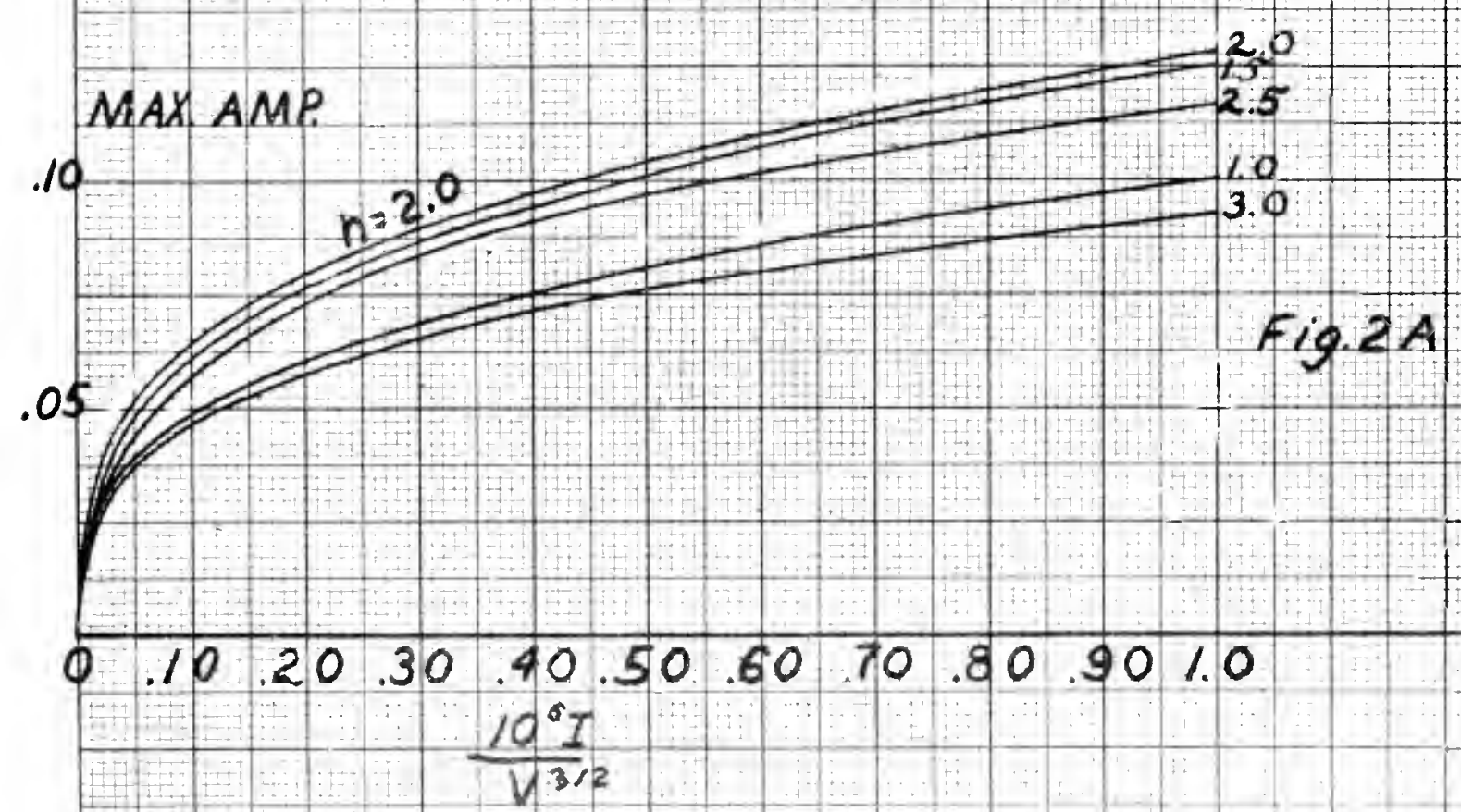





$$
\frac{b}{a}=b
$$

\section{$h=a k c o t a$}

\section{Max ama as a function of $\frac{10^{6} I}{V^{3 / z}}$}

3. MAX AMR

.25

Fig. $3 A$

$$
\begin{aligned}
& \text { - } 10.20 .30 \quad 40,50 \quad 60 \quad 70 \quad 80 \quad .9040 \\
& \frac{10^{6} I}{V^{3 / 2}}
\end{aligned}
$$





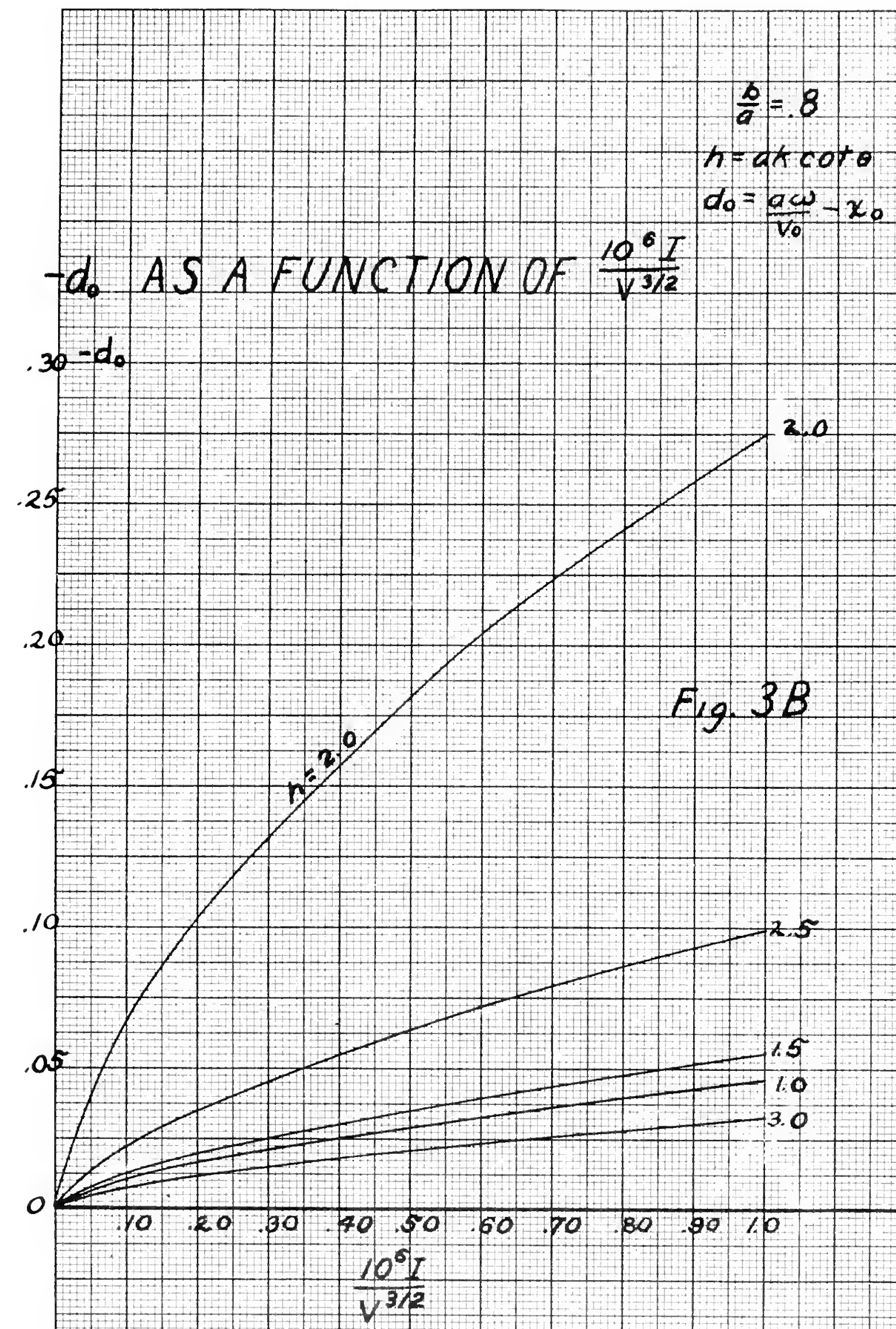


NEW YORK UNIVERSITY

INSTITUTE OF MATHEMATICAL SCIENCES

LIES:RY

4 Washington Place, View York 3. N. Y.

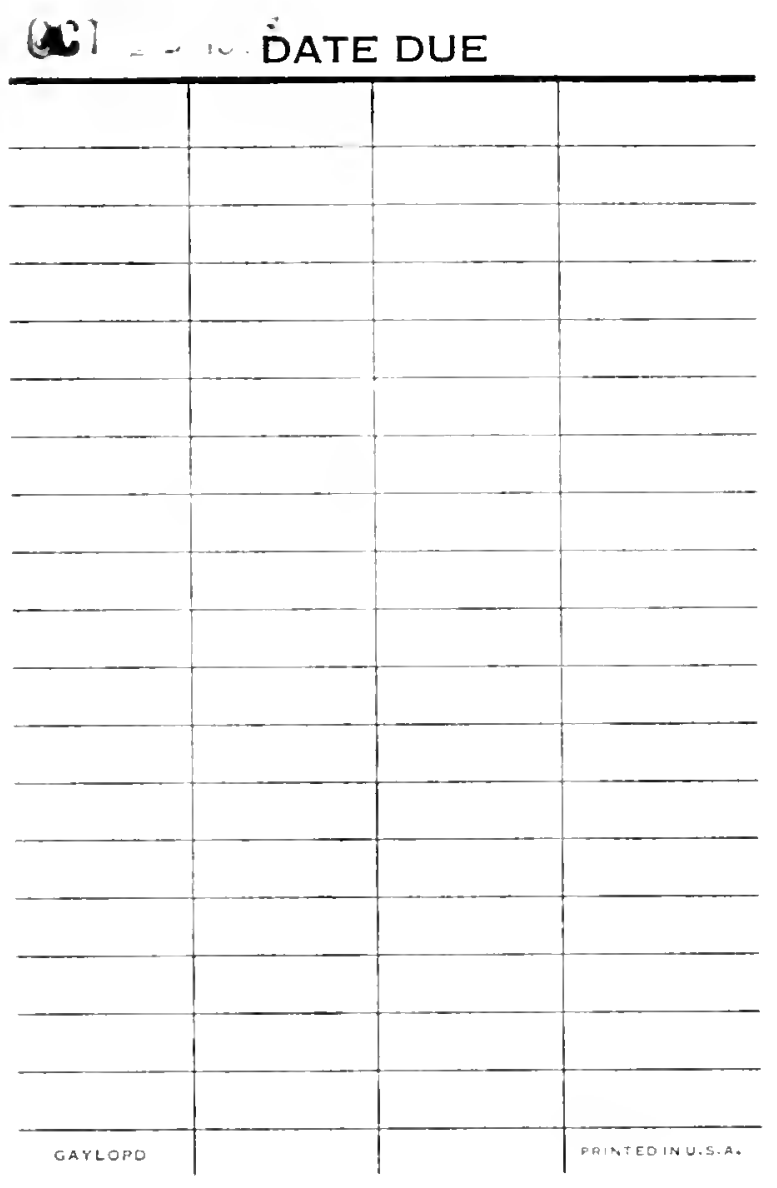




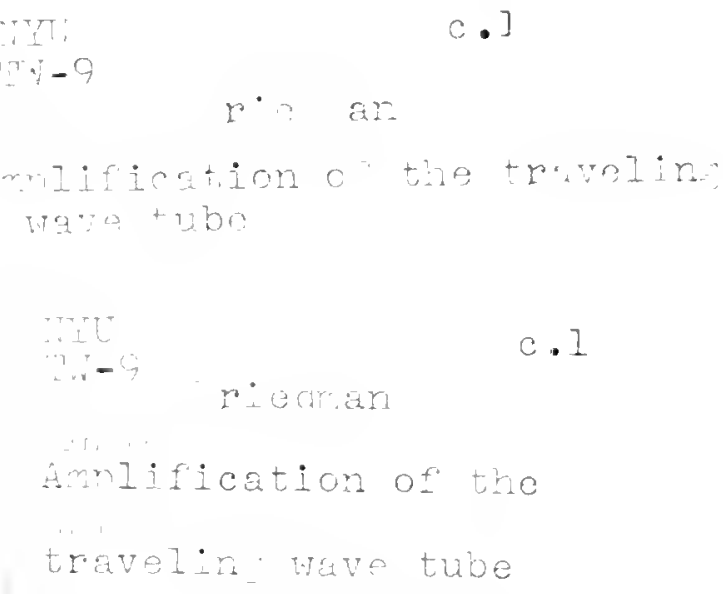

N. Y. U. Institute of Mathematical Sciences 25 Waverly Pla 5 .

New York 3, N. Y.

4 Wasnington Elace 


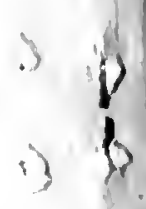

